\title{
Vagus Nerve Stimulation and Atrial Fibrillation: Revealing the Paradox
}

\author{
Rohit K. Kharbanda, MD ${ }^{1,2, a}$; Willemijn F.B. van der Does, MD ${ }^{1, a}$; \\ Lianne N. van Staveren, MD ${ }^{1}$; Yannick J.H.J. Taverne, MD, PhD ${ }^{2}$; \\ Ad J.J.C. Bogers, MD, PhD²; Natasja M.S. de Groot, MD, PhD ${ }^{1}$
}

\begin{abstract}
Background and Objective: The cardiac autonomic nervous system (CANS) plays an important role in the pathophysiology of atrial fibrillation (AF). Cardiovascular disease can cause an imbalance within the CANS, which may contribute to the initiation and maintenance of AF. Increased understanding of neuromodulation of the CANS has resulted in novel emerging therapies to treat cardiac arrhythmias by targeting different circuits of the CANS. Regarding AF, neuromodulation therapies targeting the vagus nerve have yielded promising outcomes. However, targeting the vagus nerve can be both pro-arrhythmogenic and antiarrhythmogenic. Currently, these opposing effects of vagus nerve stimulation (VNS) have not been clearly described. The aim of this review is therefore to discuss both pro-arrhythmogenic and anti-arrhythmogenic effects of VNS and recent advances in clinical practice and to provide future perspectives for VNS to treat AF.

Materials and Methods: A comprehensive review of current literature on VNS and its pro-arrhythmogenic and antiarrhythmogenic effects on atrial tissue was performed. Both experimental and clinical studies are reviewed and discussed separately.

Results: VNS exhibits both pro-arrhythmogenic and anti-arrhythmogenic effects. The anatomical site and stimulation settings during VNS play a crucial role in determining its effect on cardiac electrophysiology. Since the last decade, there is accumulating evidence from experimental studies and randomized clinical studies that low-level VNS (LLVNS), below the bradycardia threshold, is an effective treatment for AF.
\end{abstract}

Conclusion: LLVNS is a promising novel therapeutic modality to treat AF and further research will further elucidate the underlying anti-arrhythmogenic mechanisms, optimal stimulation settings, and site to apply LLVNS.

Keywords: Atrial fibrillation, neuromodulation, autonomic nervous system, electrophysiology, electropathology

Conflict of Interest: The authors reported no conflict of interest.

\section{INTRODUCTION}

The autonomic control of the heart involves a complex interplay between the cerebral cortex, brain stem, spinal cord, extra-cardiac ganglia, and intracardiac ganglia. During physiological circumstances, the cardiac autonomic nervous system (CANS) consists of a well-balanced sympathetic and parasympathetic nervous system. Cardiovascular diseases may cause an imbalance within the CANS which may contribute to initiation and maintenance of atrial tachyarrhythmias such as atrial fibrillation (AF). ${ }^{1-4}$ In patients who underwent cardiac surgery, this imbalance may also be induced by systemic inflammation, acute hemodynamic alterations, and inotropic or vasoactive drugs, which all facilitate the development of postoperative AF.,

Multiple studies in the past few decades have shown that the CANS plays an import role in cardiac arrhythmogenesis. ${ }^{1}$ Increased understanding of neuromodulation has resulted in novel emerging therapies to treat cardiac arrhythmias by targeting different circuits of the CANS. Concerning AF, neuromodulation therapies targeting the vagus nerve have yielded promising outcomes.
The vagus nerve is responsible for the parasympathetic innervation to the heart. Initial studies demonstrated that high-level vagus nerve stimulation (VNS)-inducing bradycardia-leads to pro-arrhythmogenic effects on atrial tissue. ${ }^{7,8}$ The

Address correspondence to: Natasja M.S. de Groot, MD, PhD, Department of Cardiology, Erasmus Medical Center, Rotterdam, Doctor Molewaterplein 40, 3015 GD Rotterdam, The Netherlands. Email: n.m.s.degroot@erasmusmc.nl

1 Department of Cardiology, Erasmus Medical Center, Rotterdam, The Netherlands; and

2 Department of Cardiothoracic Surgery, Erasmus Medical Center, Rotterdam, The Netherlands

${ }^{a}$ Indicates equal contribution.

For more information on author guidelines, an explanation of our peer review process, and conflict of interest informed consent policies, please see the journal's Guide for Authors.

Source(s) of financial support: N.M.S. de Groot, MD, PhD, is supported by funding grants from CVON-AFFIP (grant number 914728), NWO-Vidi (grant number 91717339), and Medical Delta. Rohit K. Kharbanda is supported by a grant from the Dutch Heart Foundation (2019T091). 
pro-arrhythmogenic effects consist of slowing of atrial conduction, shortening of the atrial effective refractory period (AERP), and increase in dispersion of atrial refractoriness. This type of VNS is commonly used to induce and maintain $\mathrm{AF}$ in experimental models. In contrast, low-level vagus nerve stimulation (LLVNS) not inducing bradycardia-has been shown to possess antiarrhythmogenic effects. ${ }^{9-11}$ Increasing evidence from preliminary clinical studies have demonstrated that LLVNS is effective in reducing the incidence and burden of (postoperative) AF. ${ }^{12-15}$

The initial success of LLVNS has resulted in the remarkable paradox that VNS can both enhance and reduce susceptibility to AF. So far, the opposing effects of high-level and low-level VNS have not been clarified. In contrast to high-level VNS, the underlying anti-arrhythmogenic effects of LLVNS have been poorly investigated. This review aims to discuss both the proarrhythmogenic and anti-arrhythmogenic effects of VNS. In particular, the underlying anti-arrhythmogenic mechanisms and recent advances in the clinical use of LLVNS will be discussed.

\section{NEURO-CARDIAC FEEDBACK}

The heart is richly innervated by the CANS, which is subdivided into an extrinsic and intrinsic part, distributed either extra-cardiac or at the level of the heart, respectively..$^{16-18}$ The intrinsic cardiac nervous system consists of a network of ganglionated plexi that contain parasympathetic and sympathetic afferent and efferent neuronal circuits with overlapping areas of influence. ${ }^{19-21}$ Sympathetic-parasympathetic interactions within the intrinsic cardiac nervous system play an important role in the control of cardiac function. ${ }^{22,23}$

Parasympathetic cardiac innervation is mediated by the vagus nerve which contains predominantly ( $80 \%)$ afferent fibers. The vagus nerve emerges from four nuclei in the medulla oblongata and contains different types of nerve fibers which are labeled as A-, Band C-group fibers, ranked by increasing degree of myelination and diameter. ${ }^{24}$ Preganglionic parasympathetic fibers synapse at autonomic ganglionic plexi (AGP) directly at the surface or inside the myocardium. In contrast, preganglionic sympathetic nerve fibers course through the intermediolateral column of the spinal cord and synapse at the extra-cardiac ganglia, including cervical and thoracic (stellate) ganglia. ${ }^{25}$ Postganglionic fibers then directly innervate the atrial and ventricular myocardium. Sympathetic nerve fibers are responsible for adrenergic cardiac stimulation. Afferent neurons carry cardio-neuro feedback from the heart toward different levels within the CANS. This neuro-cardiac feedback system is essential for the control of the sympathetic tone and parasympathetic tone of the heart and to secure a well-balanced CANS. As mentioned previously, there are also local regulatory circuits within AGPs independently regulating the sympatho-parasympathetic balance. ${ }^{17,26}$ Acute (eg, hemodynamic changes) and chronic factors (eg, cardiovascular risk factors) may cause imbalances within the CANS, which may enhance vulnerability to $A F^{1-6}$

\section{PRO-ARRHYTHMOGENIC MECHANISMS OF HIGH-LEVEL VNS}

Acetylcholine (ACh) and vasoactive intestinal peptide (VIP) are released during vagus nerve activation and are responsible for parasympathetic activation of the heart. ACh binds to muscarinic receptors (M2) that, in turn, have an inhibitory effect on G protein a-subunit. This decreases levels of cyclic adenosine monophosphate (CAMP) and protein kinase A. In addition, ACh shortens repolarization, predominantly by way of activation of the AChactivated outward potassium current (IKACh), which also impedes depolarization by inducing hyperpolarization of the resting cell membrane potential of the cardiac cell. There is an approximately linear relation between parasympathetic preganglionic discharge rates and prolongation of sinus rhythm cycle length and atrioventricular (AV) conduction time. ${ }^{27}$ Furthermore, shortening of the action potential duration consequently promotes onset and perpetuation of $\mathrm{AF}$ by reducing wavelength, which in turn facilitates reentry. ${ }^{28}$

Initial experimental studies demonstrated that administration of ACh and high-level VNS enhance AF susceptibility, particularly in combination with programmed premature atrial stimulation. ${ }^{7,29-33}$ Subepicardial injection of $A C h$ into the canine sinoatrial node (SAN) area induced a short SAN pause followed by an atrial flutter which quickly disorganized to AF. Subsequently, intravenous administration of atropine, which is a competitive inhibitor of ACh muscarinic receptors, effectively terminated AF. ${ }^{29}$ Intravenous AChinduced AF was maintained by either anti-acetylcholinesterase agents or high-level VNS. In contrast, atropine administration either slowed the fibrillatory rate or resulted in conversion to sinus rhythm. Moreover, atropine prevented subsequent AF induction by ACh administration. These results were reproduced in a larger study conducted on several animal species. ${ }^{30}$

Importantly, a higher density of IKACh has been found in the left atrium compared with the right atrium (RA). ${ }^{34}$ Arora et $\mathrm{al}^{35}$ investigated the effect of VNS and propranolol in canine hearts. Specifically, ERP shortening in response to autonomic manipulation was most outspoken in the pulmonary veins and posterior left atrium. $^{35}$

Another mechanism by which high-level VNS may initiate AF is focal activity. Haïsaguerre et $\mathrm{al}^{36}$ demonstrated that focal activity originating from the pulmonary veins and the posterior left atrium plays an essential role in AF induction. Since then, multiple underlying mechanisms for focal activity have been proposed including abnormal automaticity, triggered activity, and (transmural) micro-reentry. Emerging evidence suggests that the CANS plays an important role in focal and rotational activity originating from the pulmonary veins. ${ }^{9,37,38}$ Focal and rotational activity originating from the pulmonary veins are often co-localized with cardiac ganglionated plexi (GPs), suggesting a causal relation between altered GP activity and focal or rotational sources. ${ }^{37}$ Interestingly, ectopic activity and bursts of AF originating from the pulmonary veins could be suppressed by reflex vagal activation, eg, by way of phenylephrine injection. ${ }^{9}$ Hyperactive cardiac GPs may increase both sympathetic and parasympathetic tone, which both may initiate AF by triggered focal activity or microreentry, respectively. ${ }^{38,39}$ Multiple studies have demonstrated that sympathetic and parasympathetic co-stimulation are synergistic to the induction of atrial tachyarrhythmias. ${ }^{38-43}$ Parasympathetic firing enhances spatially heterogeneous action potential duration and dispersion in atrial refractoriness, promoting the occurrence of the re-entrant activity. Co-stimulation of the sympathetic nervous system may induce triggered activity by way of an increase in intracellular $\mathrm{Ca}^{2+}$. Thus, the combination of parasympathetic and sympathetic stimulation promotes the development of AF as triggered focal activity encounters anisotropic atrial tissue. ${ }^{44}$ 


\section{EFFECT OF HIGH-LEVEL VNS ON ATRIAL REFRACTORINESS}

As mentioned previously, high-level VNS shortens the atrial refractory period, which has been demonstrated in multiple preclinical mapping studies. For example, Hoffman et $\mathrm{al}^{7}$ performed epicardial mapping during left or right VNS to assess the relation between stimulus strength and electrophysiological changes in the atrial appendages. After vagus nerve transection, VNS was performed with different stimuli intensities defined by induced changes in AV conduction. Weak, intermediate, and maximal stimulation was defined as PR interval prolongation from 20 to 50 ms, a 2:1 AV block, and higher degrees of AV block, respectively. In these patients, activation of IKACh channels through VNS resulted in shortening of the atrial refractory period. This effect was most pronounced during maximal VNS. Whether refractoriness changed homogeneously across both atrial appendages was not examined in this study. The authors postulated that increasing the intensity of VNS results in higher concentrations of ACh released by the vagus nerve endings, thereby also gradually increasing the effect on atrial refractoriness.

Taking into consideration that the vagus nerve supply is not homogeneously distributed between both atria, it is likely that changes in refractoriness induced by VNS are nonuniformly distributed over both atria as well. Therefore, another study investigated the effect of high-level VNS on different parts of the RA. ${ }^{8}$ During VNS, large dispersion in atrial refractoriness, ranging from 50 to $140 \mathrm{~ms}$, was observed. Shortening in the refractory period was more pronounced at the inferior RA $(\Delta 100 \mathrm{~ms})$ compared with the RA appendage $(\Delta 10 \mathrm{~ms})$ and the superior RA $(\Delta 30 \mathrm{~ms})$. Interestingly, premature programmed electrical stimulation at areas with substantial shortening of the refractory period during VNS resulted more often in AF induction compared with pacing at areas that were less affected by VNS. This effect was attributed to increased local differences in the excitability of atrial cardiomyocytes.

Interestingly, in another study, it was found that this heterogeneity in response to VNS could not be attributed to heterogeneous nerve supply only, but also the heterogeneous distribution pattern of IKACh channels. ${ }^{35}$ Enhanced dispersion in atrial refractoriness during high-level VNS was confirmed in multiple studies. ${ }^{45-48}$

\section{FROM HIGH-LEVEL TO LOW-LEVEL VNS: THE TURNING POINT}

The anti-arrhythmogenic effects of VNS at lower intensity were initially observed in a canine AF model. It was demonstrated that 20 minutes of VNS, which slowed the heart rate by $<30 \%$, protected canine atria from atrial electrical remodeling (AERP shortening and enhanced AERP dispersion) during seven hours of high fixed rate atrial pacing (HFRAP). ${ }^{10}$

When the first-in-human pilot study using invasive LLVNS demonstrated that LLVNS is safe and improves functional status in patients with advanced heart failure, only a trivial effect on heart rate was observed. ${ }^{49}$ Furthermore, during a follow-up period of six months, none of these patients developed atrial tachyarrhythmias. These findings initiated a discussion on the relation between VNS intensity and its effect on heart rate and arrhythmogenicity. To elucidate the turning point from high-level to low-level VNS, Zhang et $\mathrm{al}^{11}$ examined the effect of four different VNS intensities, corresponding to $20 \%, 40 \%, 60 \%$, and $100 \%$ sinus rhythm cycle length prolongation, on AF inducibility. VNS resulting in $\leq 40 \%$ prolongation in cycle length did not increase AF inducibility and was therefore considered as an upper limit for therapeutic LLVNS modalities. ${ }^{11}$ In most experimental and clinical studies using LLVNS for its anti-arrhythmogenic effects, LLVNS is performed at an intensity lower than at which SAN or AV nodal slowing is observed.

\section{ANTI-ARRHYTHMOGENIC EFFECTS OF LOW-LEVEL VNS}

The mechanism behind the anti-arrhythmogenic effect of LLVNS has not been fully unraveled. The effect on cardiac ion channels, which will provide more insight into the anti-arrhythmogenic effects on a cellular level, is yet to be demonstrated. However, various studies have investigated the involved neurotransmitters underlying the effects of LLVNS.

As suggested by the investigators of the studies discussed in the following paragraphs, ${ }^{50,51}$ the pro-arrhythmogenic and antiarrhythmogenic effects of VNS may depend on the release of certain pro-arrhythmogenic and anti-arrhythmogenic neurotransmitters. Their release, in turn, may be dependent on the activation of specific nerve fibers. However, it could also be a matter of balance in the released neurotransmitters, with various strengths of VNS tipping the balance toward a pro-arrhythmogenic or antiarrhythmogenic effect.

The pro-arrhythmogenic effect of high-level VNS is mediated by (high concentrations of) the neurotransmitter $\mathrm{ACh}^{52}$ More recent studies also found a pro-arrhythmogenic role for the nonnoradrenergic, noncholinergic transmitter VIP. ${ }^{53-55}$

The anti-arrhythmogenic effects of LLVNS involve the nonnoradrenergic, noncholinergic transmitter nitric oxide and its signaling pathway including phosphatidylinositol-3 kinase. ${ }^{51}$ An earlier study found that the neurotransmitter vasostatin-1, when injected into GPs, exhibited anti-arrhythmogenic effects (eg, decrease in AF inducibility) and an inhibiting effect on ARGP. As these effects mimic the effects seen during LLVNS, it was hypothesized that vasostatin-1 might in part mediate the antiarrhythmogenic effect of LLVNS. ${ }^{50}$

Several studies have also investigated the effects of LLVNS on the neural activity of thoracic and cardiac GPs. An overview of these studies and their outcomes is provided in Table 1 . Sha et al ${ }^{60}$ demonstrated that baseline neural activity and SAN response during stimulation of cardiac GPs (ARGP and superior left ganglionated plexus [SLGP]) and stellate ganglia (SG) were suppressed during LLVNS, indicating that LLVNS exerts an inhibitory effect on the CANS. They postulated that the inhibitory effect on the cardiac GPs was the main anti-arrhythmogenic effect of LLVNS, as hyperactivity of the cardiac GPs contributes to AF induction. In another study, ARGP neural activity was normalized by LLVNS during HFRAP. Three hours of RAP significantly increased ARGP neural activity, but when LLVNS was added, ARGP neural activity returned to baseline values. ${ }^{57}$ The mediating effects of VNS (above the bradycardia threshold) on ARGP exhibited memory; arrhythmia induction during high-level mediastinal vagus stimulation was prevented or the induced arrhythmias were shorter for 26 minutes after cessation of stimulation. ${ }^{69}$

In contrast to these previous studies, ${ }^{57,60}$ no difference in SLGP activity was found in another study in which one week of LLVNS was performed in canines. ${ }^{58}$ A suppressing effect was only 
Table 1. Invasive and Transcutaneous LLVNS in Experimental Setting.

\begin{tabular}{|c|c|c|c|}
\hline $\begin{array}{l}\text { Author(s), } \\
\text { year }\end{array}$ & $\begin{array}{l}\text { Right, left, } \\
\text { or bilateral } \\
\text { VNS }\end{array}$ & $\begin{array}{l}\text { Frequency } \\
\text { Pulse width } \\
\text { Waveform } \\
\text { On-off/Continuous }\end{array}$ & Intensity \\
\hline \multicolumn{4}{|l|}{ Invasive LLVNS } \\
\hline $\begin{array}{l}\text { Li et } a l_{,}^{56} \\
2009\end{array}$ & $R+L$ & $\begin{array}{l}20 \mathrm{~Hz} \\
100 \mu \mathrm{s} \\
\text { Square } \\
\text { Continuous }\end{array}$ & $\begin{array}{l}1 \mathrm{~V} \text { below voltage } \\
\text { slowing SAN/AVN }\end{array}$ \\
\hline$Y u_{1}^{57} 2011$ & $R+L$ & $\begin{array}{l}20 \mathrm{~Hz} \\
100 \mu \mathrm{s} \\
\text { Square } \\
\text { Continuous }\end{array}$ & $\begin{array}{l}10 \% \text { and } 50 \% \text { below } \\
\text { voltage slowing } \\
\text { SAN/AVN }\end{array}$ \\
\hline $\begin{array}{l}\text { Shen et al, }{ }^{58} \\
2011\end{array}$ & L & $\begin{array}{l}13 \mathrm{~Hz} \\
450 \mu \mathrm{s} \\
\mathrm{NA} \\
\text { Continuous }\end{array}$ & $\begin{array}{l}1 \mathrm{~V} \text { below voltage } \\
\text { decreasing } H R>20 \%\end{array}$ \\
\hline $\begin{array}{l}\text { Sheng } \\
\text { et } \mathrm{al}_{1}^{59} \\
2011\end{array}$ & $R+L$ & $\begin{array}{l}20 \mathrm{~Hz} \\
100 \mu \mathrm{s} \\
\text { Square } \\
\text { Continuous }\end{array}$ & $\begin{array}{l}10 \% \text { or } 50 \% \text { below } \\
\text { voltage slowing } \\
\text { SAN/AVN }\end{array}$ \\
\hline $\begin{array}{l}\text { Sha et al, } \\
2011\end{array}$ & $\mathrm{R}$ & $\begin{array}{l}20 \mathrm{~Hz} \\
100 \mu \mathrm{s} \\
\mathrm{NA} \\
\text { Continuous }\end{array}$ & $\begin{array}{l}50 \% \text { below voltage } \\
\text { slowing SAN/AVN }\end{array}$ \\
\hline $\begin{array}{c}\text { Yu et } \mathrm{al}_{1}^{57} \\
2012\end{array}$ & $\mathrm{R}$ & $\begin{array}{l}20 \mathrm{~Hz} \\
100 \mu \mathrm{s} \\
\text { Square } \\
\text { Continuous }\end{array}$ & $\begin{array}{l}50 \% \text { below voltage } \\
\text { slowing SN/AVN }\end{array}$ \\
\hline $\begin{array}{l}\text { Shen et al, } \\
2013\end{array}$ & L & $\begin{array}{l}15 \mathrm{~Hz} \\
500 \mu \mathrm{s} \\
\text { Square } \\
\text { On (60 s) - off (12 s) }\end{array}$ & $\begin{array}{l}1 \mathrm{~mA} \text { below stimulus } \\
\text { strength }(\mathrm{mA}) \\
\text { decreasing heart rate } \\
>20 \%\end{array}$ \\
\hline
\end{tabular}

Duration stimulation at PV and atrial appendage sites during measurements 3 h i.c.w. high-frequency ARGP + SLGP stimulation during measurements

Group 1: $7 \mathrm{~d}$ continuous Group 2: $3 \times 1 \mathrm{~d}$ per $7 \mathrm{~d}$ (sham or active), other days HFRAP. total $21 \mathrm{~d}$

Group 1.3 h i.c.w. HFRAP (after $3 \mathrm{~h}$ only HFRAP) Group $2.6 \mathrm{~h} \mathrm{i.cw.}$ HFRAP

3 h i.c.w. high-frequency stimulation and pacing at various atrial sites,

SLGP and (R)SG

3 h i.c.w. HFRAP (after $3 \mathrm{~h}$ only HFRAP)

AERP normalization 2. WOV normalization

Group $1.7 \mathrm{~d}$

Group 2. Control

Cardiac

AF threshold $\uparrow$

Suppression of 1.AERP $\downarrow$ + dispersion $\uparrow$

2. WOV $\uparrow$

Group 2:

PATs $\downarrow$

AERP normalization/

unchanged

2. WOV normalization

unchanged

3. AFCL $\uparrow$

4. AF duration $\downarrow$

AF threshold $\uparrow$

None $\downarrow$ SLGP function

2. $\downarrow$ (R)SG function*

3. $\downarrow$ ARGP NA

ARGP neural activity normalization

None

Suppression of AF induced by cholinergic stimulation

None

$\uparrow$ SK2 proteins 2. $\uparrow$ SK2 protein expression in cell membrane

3. $\uparrow \mathrm{TH}$ - ganglion cells (most were ChAT+) 
Table 1. Continued

\begin{tabular}{|c|c|c|c|c|c|c|c|}
\hline \multirow{3}{*}{$\begin{array}{l}\text { Author(s), } \\
\text { year }\end{array}$} & \multicolumn{2}{|r|}{ Frequency } & \multirow[b]{3}{*}{ Intensity } & \multirow[b]{3}{*}{ Duration } & \multirow[b]{3}{*}{ Cardiac } & \multirow[b]{2}{*}{ Outcomes } & \multirow[b]{3}{*}{ Other } \\
\hline & $\begin{array}{l}\text { Right, left, } \\
\text { or bilateral }\end{array}$ & $\begin{array}{l}\text { Pulse width } \\
\text { Waveform }\end{array}$ & & & & & \\
\hline & VNS & On-off/Continuous & & & & Neural measurements & \\
\hline $\begin{array}{l}\text { Stavrakis } \\
\text { et al, } \\
2013\end{array}$ & $\mathrm{R}$ & $\begin{array}{l}20 \mathrm{~Hz} \\
100 \mu \mathrm{s} \\
\text { Square } \\
\text { Continuous }\end{array}$ & $\begin{array}{l}\text { 10\% below voltage slow- } \\
\text { ing SAN/AVN }\end{array}$ & $\begin{array}{l}3 \text { h i.c.w. HFRAP (after } 3 \mathrm{~h} \\
\text { only HFRAP) }\end{array}$ & $\begin{array}{l}\text { 1. Duration ACh-induced } \\
\text { AF } \downarrow \\
\text { 2. WOV normalization }\end{array}$ & ARGP function* $\downarrow$ & $\begin{array}{l}\text { NO inhibitors abol- } \\
\text { ished effects of } \\
\text { LLVNS }\end{array}$ \\
\hline $\begin{array}{l}\text { Liu et } \mathrm{al}_{1}^{54} \\
2013\end{array}$ & $R+L$ & $\begin{array}{l}20 \mathrm{~Hz} \\
100 \mu \mathrm{s} \\
\text { Square } \\
\text { Continuous }\end{array}$ & $\begin{array}{l}\text { 50\% below voltage pro- } \\
\text { longing SR CL } 30 \text { ms or } \\
\text { less }\end{array}$ & $3 \mathrm{~h}+\mathrm{HFRAP}$ & $\begin{array}{l}\text { 1. AERP—no effect } \\
\text { dispersion—no effect } \\
\text { 2. WOV unchanged }\end{array}$ & None & None \\
\hline Cho, 2014 & $\mathrm{R}$ & $\begin{array}{l}20 \mathrm{~Hz} \\
200 \mu \mathrm{s} \\
\text { Square } \\
\text { Continuous }\end{array}$ & $\begin{array}{c}40 \% \text { below voltage } \\
\text { decreasing HR }\end{array}$ & $\begin{array}{l}\text { Group 1. } 3 \mathrm{~h}+\text { HFRAP } \\
\text { Group 2. Control }\end{array}$ & $\begin{array}{l}\text { 1. AERP unchanged } \\
\text { 2. WOV unchanged } \\
\text { 3. AF inducibility } \\
\text { unchanged }\end{array}$ & None & $\begin{array}{l}\text { No difference in nerve } \\
\text { density and sprout- } \\
\text { ing evaluated by } \\
\text { anti-TH and anti- } \\
\text { growth associated } \\
\text { protein-43 staining }\end{array}$ \\
\hline $\begin{array}{l}\text { Yuan et al, }{ }^{63} \\
2015\end{array}$ & $\mathrm{~L}$ & $\begin{array}{l}1 \mathrm{~Hz} \\
40 \mathrm{~ms} \\
\text { Rectangular } \\
\text { Continuous }\end{array}$ & $2 \mathrm{~V}$ & $\begin{array}{l}\text { Group } 1.4 \text { wk i.c.w. HFRAP } \\
\text { Group 2. Only RAP }\end{array}$ & $\begin{array}{l}\text { 1. Incidence sustained } \\
\text { AF } \downarrow\end{array}$ & None & $\begin{array}{l}\text { 1. GAP43 nerve } \\
\text { density } \downarrow \\
\text { 2. TH nerve } \\
\text { density } \downarrow\end{array}$ \\
\hline $\begin{array}{c}\text { Lu et } \mathrm{al}_{,}^{64} \\
2016\end{array}$ & $\mathrm{R}$ & $\begin{array}{l}20 \mathrm{~Hz} \\
100 \mu \mathrm{s} \\
\text { Square } \\
\text { Continuous }\end{array}$ & $\begin{array}{l}\text { 50\% below voltage slow- } \\
\text { ing SAN or second } \\
\text { degree AV block }\end{array}$ & $\begin{array}{l}\text { Group } 1.12 \text { h i.c.w. HFRAP } \\
\text { Group 2. Only HFRAP } \\
\text { Group 3. Control }\end{array}$ & AERP—no effect & None & $\begin{array}{l}\text { 1. ARGP expression of } \\
\text { nerve growth factor } \\
\text { and neurturin } \downarrow \\
\text { 2. glycogen } \\
\text { accumulation } \downarrow\end{array}$ \\
\hline \multicolumn{8}{|c|}{ Transcutaneous LLVNS } \\
\hline $\begin{array}{l}\text { Yu et al, } \\
2013\end{array}$ & $\mathrm{R}$ & $\begin{array}{l}20 \mathrm{~Hz} \\
1000 \mu \mathrm{s} \\
\text { Square } \\
\text { Continuous }\end{array}$ & $\begin{array}{l}80 \% \text { below voltage slow- } \\
\text { ing SAN/AVN }\end{array}$ & $\begin{array}{l}3 \text { h i.c.w. HFRAP (after } 3 \mathrm{~h} \\
\text { only HFRAP) }\end{array}$ & $\begin{array}{l}\text { 1. AERP normalization } \\
\text { 2. WOV normalization }\end{array}$ & $\begin{array}{l}\text { ARGP neural activity } \\
\text { normalization }\end{array}$ & None \\
\hline $\begin{array}{l}\text { Chen et al, } \\
2015\end{array}$ & $\mathrm{R}$ & $\begin{array}{l}20 \mathrm{~Hz} \\
1000 \mu \mathrm{s} \\
\text { Square } \\
\text { Continuous }\end{array}$ & $\begin{array}{l}80 \% \text { below voltage slow- } \\
\text { ing SAN/AVN }\end{array}$ & $\begin{array}{l}\text { Group } 1.9 \text { h i.c.w. HFRAP } \\
\text { Group 2. Only HFRAP } \\
\text { Group 3. Control }\end{array}$ & $\begin{array}{l}\text { 1. AF duration } \downarrow \\
\text { 2. AFCL } \uparrow\end{array}$ & None & $\begin{array}{l}\mathrm{Cx} 40 \uparrow \\
\mathrm{Cx} 43 \uparrow\end{array}$ \\
\hline $\begin{array}{l}\text { Chen et al, } \\
2015\end{array}$ & $\mathrm{~L}$ & $\begin{array}{l}20 \mathrm{~Hz} \\
1000 \mu \mathrm{s} \\
\text { Square } \\
\text { Continuous }\end{array}$ & $\begin{array}{l}\text { 80\% below voltage slow- } \\
\text { ing SAN/AVN }\end{array}$ & $\begin{array}{l}\text { Group } 1.9 \text { h i.c.w. HFRAP } \\
\text { Group 2. Only HFRAP } \\
\text { Group 3. Control }\end{array}$ & $\begin{array}{l}\text { 1. AF duration } \downarrow \\
\text { 2. AFCL } \uparrow \\
\text { 3. AERP + AERP disper- } \\
\text { sion unchanged } \\
\text { 4. WOV unchanged }\end{array}$ & None & $\begin{array}{l}\mathrm{Cx} 40 \uparrow \\
\mathrm{Cx} 43 \uparrow\end{array}$ \\
\hline
\end{tabular}


observed on left SG nerve activity, although SLGP activity remained unchanged. In the group with HFRAP and left LLVNS, similar effects on neural activity were found. In addition, LLVNS reduced the density of tyrosine hydroxylase-positive nerves in the SG, which could explain the reduced SG nerve activity. In a subsequent study, a higher expression of small-conductance calcium-activated potassium channels and proteins was found in the cell membrane of the SG of canines who underwent LLVNS for one week. ${ }^{61}$ Smallconductance calcium-activated potassium channels are responsible for slow afterhyperpolarization, which hyperpolarizes neurons and thus suppresses nerve activity from the SG.

The investigators attributed the difference in outcomes to the duration of stimulation, proposing that short-term LLVNS does suppress nerve activity at cardiac GPs, yet this effect does not sustain with long-term LLVNS. However, there are some differences in methodology between this study ${ }^{58}$ and the previously mentioned studies, ${ }^{57,60}$ including the duration of LLVNS (three hours vs seven days) and also the site and settings of LLVNS (right or both vs left, $20-30 \mathrm{~Hz}$ vs $13 \mathrm{~Hz}$ ) differed. Finally, in their longterm study, no anesthetics were used during neural measurements and LLVNS.

Only a few studies examined changes in neural-cardiac activity during noninvasive transcutaneous LLVNS (tLLVNS). One study performed recordings of ARGP in canines during three hours of HFRAP followed by three hours of tLLVNS at the right tragus. ${ }^{65}$ Anterior right GP activity increased during HFRAP and returned to baseline values during tLLVNS, which was set at $80 \%$ of the threshold voltage required to slow SAN depolarization rate or AV nodal conduction. A causal relation was demonstrated, as the effect of tLLVNS was abolished after transection of the vagus nerve. This effect on ARGP activity in a tLLVNS model is similar to the outcomes during acute models with invasive LLVNS. ${ }^{57,60}$

Clancy et $\mathrm{al}^{69}$ investigated the effects of tLLVNS $(30 \mathrm{~Hz}$, sensory threshold) on cardiovascular autonomic function in healthy volunteers. In 34 participants, they found improved heart rate variability with a shift toward vagal dominance during 15 minutes of tLLVNS. This study was the first clinical study to demonstrate that transcutaneous LLVNS changes the autonomic balance toward a vagal dominance.

\section{EXPERIMENTAL AND CLINICAL APPLICATION OF INVASIVE LLVNS}

Multiple experimental and clinical studies have been performed to assess the anti-arrhythmogenic properties of invasive LLVNS. In 2009, Li et al $^{56}$ reported that three hours of bilateral LLVNS in anesthetized canines was effective in decreasing AF susceptibility. High-frequency stimulation ( $40 \mathrm{~ms}$ train of VNS) was performed 2 $\mathrm{ms}$ after premature atrial stimulation at the pulmonary veins and both atrial appendages. After LLVNS, the AF threshold, defined as the lowest voltage of high-frequency stimulation that induced AF, was increased. However, the increase was unequally distributed in the different atrial sites, highlighting that the CANS is heterogeneously spread over the atria. After transection of both cervical vagosympathetic trunks, subsequent stimulation at the distal ends of the vagus nerve trunks did not change study outcomes. This indicates that the anti-arrhythmogenic effect of LLVNS was mostly dependent on the activation of efferent vagus nerve fibers. However, minimal differences in anti-arrhythmogenic effects between stimulation before and after transection indicate the interaction between afferent and efferent fibers.

Subsequent studies confirmed the anti-arrhythmogenic effect of invasive LLVNS and also provided evidence that atrial electrical remodeling (AERP shortening and enhanced AERP dispersion), which occurs during HFRAP, was inhibited, or even reversed by LLVNS (Table 1). Sheng et $\mathrm{al}^{59}$ investigated the effect of LLVNS during AF. In anesthetized canines, AF was induced with strong cholinergic stimulation by applying $A C h$ into the anterior right ganglionated plexus (ARGP) or the RA appendage. Bilateral invasive LLVNS $(20 \mathrm{~Hz}, 10 \%-50 \%$ below threshold slowing SAN depolarization rate or $\mathrm{AV}$ conduction) prolonged $\mathrm{AF}$ cycle length and shortened the duration of AF episodes. ${ }^{59}$

Interestingly, LLVNS has also been shown to inhibit structural remodeling. In comparison with canines with only HFRAP, 12 hours of HFRAP in combination with right LLVNS $(20 \mathrm{~Hz}, 50 \%$ below voltage slowing SAN depolarization rate or inducing second degree AV block) inhibited glycogen accumulation, ${ }^{64}$ a biomarker for structural remodeling. ${ }^{70}$

Yuan et $\mathrm{al}^{63}$ demonstrated that tachycardia-induced autonomic remodeling could be prevented by LLVNS. Histological examination of the rabbit atria was performed after four weeks of HFRAPinduced persistent $A F$ and compared with rabbit atria receiving simultaneous LLVNS (rectangular pulses, $40 \mathrm{~ms}$ duration at $1 \mathrm{~Hz}$, $2 \mathrm{~V}$ ). Tyrosine hydroxylase and growth-associated protein 43 densities were significantly lower in the LLVNS group, indicating less sympathetic nerve sprouting. In line with these findings, Beaumont et $\mathrm{al}^{71,72}$ demonstrated that remodeling in the CANS induced by pressure overload or myocardial infarction can be mitigated by chronic VNS. The possibility to suppress autonomic remodeling by LLVNS was further substantiated during HFRAP, ${ }^{57}$ confirming that LLVNS may indeed be a promising strategy to break the vicious cycle of "AF begets AF." The various ways through which LLVNS may break this cycle are depicted in Figure 1.

In a clinical setting, LLVNS reduced levels of postoperative inflammatory markers associated with AF after cardiac surgery. As demonstrated in Table 2, Rossi et al $^{73}$ performed invasive LLVNS (50 $\mathrm{Hz}$, PR interval prolongation of $\geq 30 \%$ ) in a pilot study with 35 patients who underwent cardiac surgery (20 active, 15 control) and evaluated the levels of postoperative inflammatory markers. A temporary pacemaker wire, which was stitched at the inferior vena cava-inferior ganglionated plexus, was used to perform neurostimulation at the intensive care unit. After six hours, the stimulation group had significantly lower levels of interleukin 6, tumor necrosis factor alpha, vascular endothelial growth factor, and epidermal growth factor. Postoperative AF occurred in $7 \%$ and $25 \%$ in the stimulation group and control group, respectively.

Subsequently, Stavrakis et al $^{12}$ applied invasive LLVNS as a novel approach to prevent postoperative AF. Patients who underwent cardiac surgery received a temporary pacemaker wire at the preganglionic vagus nerve fibers alongside the lateral aspect of the superior vena cava. A total of 54 patients were randomized to $72-$ hours of active LLVNS $(20 \mathrm{~Hz}, 50 \%$ below the threshold for a significant slowing of SAN depolarization rate) or sham ( $n=26$ vs $n=$ 28 , respectively). Postoperative AF occurred less frequently in the LLVNS compared with the sham controls $(12 \%$ vs $36 \%, p=0.027)$ and was partly explained by attenuation of the inflammation response (lower levels of tumor necrosis factor alpha and interleukin 6). These anti-inflammatory effects have also been found during transcutaneous LLVNS (tLLVNS), possibly revealing a simple, noninvasive intervention to prevent postoperative AF. ${ }^{13}$ The vagus 
Targets for LLVNS to break the AF cycle

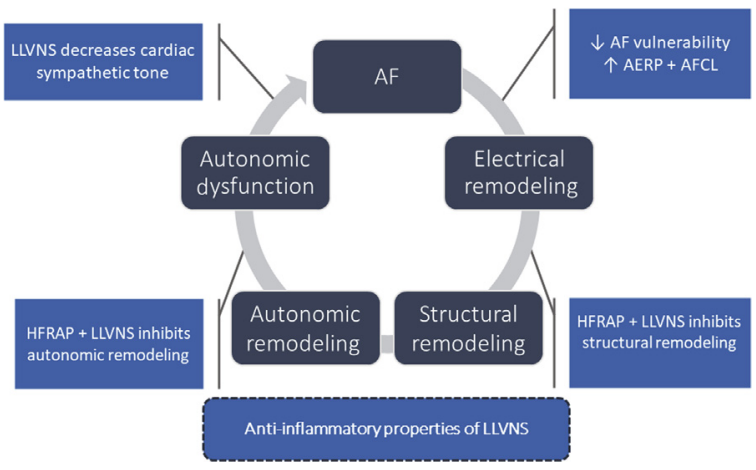

Figure 1. The various pathways are depicted by which LLVNS may inhibit the occurrence and continuation of the cycle in which "AF begets AF." AFCL, atrial fibrillation cycle length; LLVNS, low-level vagal nerve stimulation; $\uparrow$, higher; $\downarrow$, lower. [Color figure can be viewed at www.neuromodulationjournal.org]

nerve attenuates the inflammatory response by activation of the cholinergic anti-inflammation pathway. ${ }^{74,75}$ Although the outcomes seem promising, at present, there are no randomized controlled trials investigating the efficacy of ( $t$ )LLVNS in preventing inflammation-related complications after cardiac surgery.

\section{EXPERIMENTAL AND CLINICAL APPLICATION OF TRANSCUTANEOUS LLVNS}

The sensible auricle branch of the vagus nerve, which is distributed to the skin of the external acoustic meatus and the auricle, serves as the target for tLLVNS. In 14 ears of seven cadavers, these vagal nerve endings were most frequently found at the cymba conchae, antihelix, tragus, and cavity of the concha. ${ }^{76}$ Although some inconsistencies have been found in this study, ${ }^{77}$ functional magnetic resonance imaging studies have reported activity in afferent vagus nerve projection sites during tLLVNS at the left tragus and cymba conchae. ${ }^{78}$ The auricle branch of the vagus nerve contains solely vagus nerve afferents. This may serve as an advantage for tLLVNS compared with LLVNS, because the vagus nerve contains sympathetic nerve fibers that are also stimulated during invasive LLVNS, although tLLVNS stimulates solely afferent fibers at the earlobe which then relay their action through a mostly unknown vagal pathway. ${ }^{79}$ In contrast, much is unknown on the mechanisms of LLVNS. For example, the importance and effect of (contralateral) afferent-efferent interactions in LLVNS is largely unknown, thus we cannot predict all consequences. ${ }^{80}$

The efficacy of tLLVNS was established in several experimental and clinical studies. Yu et $\mathrm{al}^{65}$ were the first to perform tLLVNS $(20 \mathrm{~Hz}, 80 \%$ below bradycardia threshold) in an AF canine model $(n=6)$. After three hours of HFRAP, progressive shortening and enhanced dispersion in ERP were observed. Subsequently, three hours of right tLLVNS reversed these changes. This protective effect was not observed in canines who underwent bilateral vagus nerve transection, again implying an important role of efferent vagus nerve fibers in the anti-arrhythmogenic effects of LLVNS. tLLVNS did not affect AF cycle length or duration. In another study performed in 32 canines, a shorter duration of AF was observed after nine hours of HFRAP including left or right tLLVNS $(20 \mathrm{~Hz}, 80 \%$ below threshold slowing SAN depolarization rate or AV conduction) compared with controls who received RAP only. ${ }^{66,67}$ The authors proposed that the anti-arrhythmogenic effects of tLLVNS are not exclusive to right VNS. As tLLVNS also prevented RAP-induced reduction in connexin expression, the anti-arrhythmogenic effects of tLLVNS were partly attributed to inhibition of structural remodeling.

At present, three randomized clinical trials have been published examining the anti-arrhythmogenic efficacy of tLLVNS. In the first study, 40 patients scheduled for catheter ablation of paroxysmal AF were randomized. ${ }^{13}$ Under general anesthesia, baseline AERP, and AF cycle length measurements were performed after inducing AF. Subsequently, patients either received one hour of right tLLVNS (20 $\mathrm{Hz}, 50 \%$ below threshold slowing sinus rate or prolonging atrial-his interval) or sham control (ear lobe) (20 vs 20 patients). After one hour, AF was induced for a second time and the measurements were repeated. More attempts were required to induce $A F$ and $A F$ duration was shorter in patients who received tLLVNS. Atrial ERP and AF cycle length were prolonged in the tLLVNS group. In addition, one hour of tLLVNS acted anti-inflammatory by decreasing tumor necrosis factor alpha and C-reactive protein levels.

In the second study, patients were randomized to tLLVNS via the triangular fossa or sham (sham device) after cardiac surgery. ${ }^{14}$ Patients who received tLLVNS were less likely to develop postoperative AF compared with controls ( $20 \%$ vs $55 \%, p=0.02$ ).

In the third study, Stravrakis et $\mathrm{al}^{15}$ examined the effect of tLLVNS on AF burden in ambulatory patients with paroxysmal AF. During six months, patients received either tLLVNS via the ear lobe (sham) or tragus (active arm) for one hour per day. Two weeks of continuous rhythm monitoring was performed at baseline after three and six months. A substantial decrease in AF burden of $85 \%$ was observed in the active $\operatorname{arm}(p=0.01$, confidence interval $[\mathrm{Cl}]=$ 0.03-0.65). In addition, long-term tLLVNS exhibited antiinflammatory effects; tumor necrosis factor alpha levels were lowered at six months compared with baseline.

Experimental and clinical studies on LLVNS for the treatment of AF are listed in Table 1 and 2, respectively. Thus, in recent years various clinical studies have confirmed tLLVNS to be a safe and promising therapeutic procedure for AF.

\section{CONSIDERATIONS REGARDING LLVNS STIMULATION SETTINGS}

The difference between anti-arrhythmogenic LLVNS and proarrhythmogenic high-level VNS is partly based on the stimulation intensity, which corresponds to stimulation of different vagus nerve fibers. As mentioned earlier, high-level VNS is performed with an intensity above the bradycardia threshold; bradycardia indicates stimulation of smaller myelinated slow B-fibers and/or unmyelinated C-fibers. Low-level VNS is set below the bradycardia threshold, thereby stimulating the larger and myelinated A-fibers and fast B-fibers. $^{81,82}$

Importantly, LLVNS settings-frequency, pulse width, amplitude, and waveform-are not completely uniform throughout the previously discussed studies. The stimulation of specific vagus nerve fibers depends on pulse intensity; a function of pulse width and output current. ${ }^{83}$ Activation thresholds for the various vagus nerve fibers by stimulating the right cervical vagus nerve were examined in canine atria. The activation threshold for slow B-fibers $(4.4 \pm 0.5$ 
Table 2. Invasive and Transcutaneous LLVNS for Atrial Fibrillation in Humans.

\begin{tabular}{|c|c|c|c|c|c|c|}
\hline Author(s), year & Location & $\begin{array}{l}\text { Frequency } \\
\text { Pulse width } \\
\text { Waveform }\end{array}$ & \multicolumn{2}{|l|}{ VNS settings } & \multicolumn{2}{|r|}{ Outcomes } \\
\hline \multicolumn{7}{|l|}{ Invasive LLVNS } \\
\hline Rossi et al, ${ }_{1}^{73} 2012^{*}$ & $\begin{array}{l}\text { IVC-IAGP } \\
+ \\
\text { R-VN }\end{array}$ & $\begin{array}{l}50 \mathrm{~Hz} \\
1000 \mu \mathrm{s} \\
\mathrm{NA}\end{array}$ & $\begin{array}{l}\text { At intensity resulting in PR } \\
\text { interval } \geq 30 \%\end{array}$ & $6 \mathrm{~h} /$ train duration $180 \mathrm{~ms}$ & POAF-no effect & $\begin{array}{c}\text { TNF- } a \downarrow \\
I L-6 \downarrow \\
\text { VEGF } \downarrow \\
\text { EGF } \downarrow\end{array}$ \\
\hline Stavrakis et al, ${ }^{12} 2017^{*}$ & $\mathrm{R}-\mathrm{VN}$ & $\begin{array}{l}20 \mathrm{~Hz} \\
100 \mu s \\
\mathrm{NA}\end{array}$ & $\begin{array}{l}\text { 50\% below threshold } \\
\text { slowing SAN }\end{array}$ & $72 \mathrm{~h}$ & POAF $\downarrow$ & $\begin{array}{r}\text { TNF- } a \downarrow \\
I L-6 \downarrow\end{array}$ \\
\hline \multicolumn{7}{|l|}{ Transcutaneous LLVNS } \\
\hline Stavrakis et al, ${ }^{13} 2015$ & $\mathrm{R}-\mathrm{VN}$ & $\begin{array}{l}20 \mathrm{~Hz} \\
\quad 1000 \text { us square }\end{array}$ & $\begin{array}{l}\text { 50\% below threshold } \\
\text { slowing SAN or pro- } \\
\text { longing AH interval }\end{array}$ & $1 \mathrm{~h}$ & $\begin{array}{c}\text { AF duration } \downarrow \\
\text { AFCL } \uparrow \\
\text { AERP } \uparrow\end{array}$ & $\begin{array}{r}\text { TNF- } a \downarrow \\
\text { CRP } \downarrow\end{array}$ \\
\hline Andreas et al, ${ }^{14} 2019^{*}$ & NA & $\begin{array}{l}1 \mathrm{~Hz} \\
\mathrm{NA} \\
\mathrm{NA}\end{array}$ & $1 \mathrm{~mA}$ & $\begin{array}{l}40 \text { ms (LLVNS) - } 20 \text { ms } \\
\text { (pause) } \\
\text { Up to } 2 \text { wk after surgery }\end{array}$ & POAF $\downarrow$ & $\begin{array}{l}\text { CRP_no effect } \\
\text { IL-6_-no effect }\end{array}$ \\
\hline Stavrakis et al, ${ }^{15} 2020^{*}$ & $\mathrm{R}-\mathrm{VN}$ & $\begin{array}{l}20 \mathrm{~Hz} \\
200 \mu s \\
\text { NA }\end{array}$ & $\begin{array}{l}1 \mathrm{~mA} \text { below discomfort } \\
\text { threshold }\end{array}$ & 1h per day for 6 mo & $\begin{array}{c}\text { AF burden } \downarrow \\
\text { HRV } \downarrow\end{array}$ & TNF- $a \downarrow$ \\
\hline
\end{tabular}

AFCL, atrial fibrillation cycle length; AH interval, atrial-His bundle interval; CRP, C-reactive protein; EGF, epidermal growth factor; HRV, heart rate variability; IVCIAGP, inferior vena cava-inferior atrial ganglionated plexus; NA, not available; POAF, postoperative atrial fibrillation; R-VN, right vagus nerve; TNF-a, tumor necrosis factor alpha; VEGF, vascular endothelial growth factor.

*Post-cardiac surgery setting.

$\mathrm{mA})$, which induced bradycardia, ${ }^{84}$ was eight times higher than the threshold for A-fibers $(0.52 \pm 0.08 \mathrm{~mA})$ and almost three times higher than the threshold for fast B-fiber $(1.5 \pm 0.2 \mathrm{~mA})$ activation. $^{82}$ Based on these observations, the slight variations in settings in the previously mentioned LLVNS studies do not seem to be of great importance. The lowest stimulation intensity used during invasive LLVNS is $50 \%$ below the bradycardia threshold; therefore, it is likely that A-fibers and fast B-fibers are stimulated in these studies.

The frequency setting seems to mainly influence the strength of the effect exerted by the stimulated fibers. ${ }^{82}$ Interestingly, two studies on invasive LLVNS and tLLVNS found that high pulse intensity and low frequency selectively activated C-fibers. ${ }^{78,81}$ The upper limit for frequency during invasive LLVNS is set to be $50 \mathrm{~Hz}$, as irreversible damage to the vagus nerve after continuous stimulation at higher frequencies was found. ${ }^{24}$ The various waveforms of stimulation can be used to increase energy efficiency or increase stimulation selectivity. ${ }^{83}$

Although various settings seem to be effective, the exact underlying anti-arrhythmogenic effect of LLVNS on human atria remains largely unknown. However, these settings might become of importance to optimize the therapeutic effect of LLVNS. Furthermore, it is also unknown whether stimulation settings used during invasive LLVNS can be translated to tLLVNS. Transcutaneous LLVNS using the sensible auricle branch of the vagus nerve only stimulates afferent vagus nerve A-fibers, which then relay a response to the nucleus tractus solitarius. ${ }^{85}$ Although direct stimulation at the vagus nerve also activates efferent fibers coursing toward the heart. It is therefore most likely that VNS stimulation settings are dependent on the anatomical stimulation target site and may differ between patients. Future research is therefore warranted to investigate the different stimulation settings at different anatomical target sites and correlate this with electrophysiological changes in the atria.

\section{FUTURE PERSPECTIVES FOR LLVNS TO TREAT AF}

The switch from invasive to noninvasive LLVNS for the treatment of AF was an important step. The noninvasive methodology provides us the possibility to further unravel, develop, and implement this promising treatment into clinical practice. Clinical studies using tLLVNS have shown promising results such as reduction in the incidence of postoperative $A F$, a lower $A F$ burden, and suppression of the inflammatory response. However, to establish an evidence-based method of LLVNS for the treatment of cardiac arrhythmias, a better comprehension of the exact underlying neurological and anti-arrhythmogenic pathways is needed. More extensive research on optimal stimulation settings as well as short- and long-term outcomes is essential. Furthermore, the selection of the correct VNS target for each patient is important, as the anatomical distribution of the vagal nerve branches may vary between patients.

To optimize LLVNS, Napadow et $a^{86}$ aimed to synchronize tLLVNS with respiration. During respiratory-gated auricular VNS, stimuli are only delivered during expiration, as the vagal outflow is inhibited during inspiration by a feedback loop which causes the well-known respiratory sinus arrhythmia. ${ }^{86}$ In this way, stimulation may be more effective, as it is ineffective to stimulate during a period in which vagal outflow will be inhibited. Innovations such as these may be useful in optimizing the anti-arrhythmogenic effect of LLVNS. Currently, it can be concluded that LLVNS is a promising 
novel therapeutic modality for AF and further research will further elucidate the mechanisms behind the anti-arrhythmogenic effects of LLVNS.

\section{Authorship Statements}

Rohit K. Kharbanda, Natasja M.S. de Groot, and Ad J.J.C. Bogers designed the study. Rohit K. Kharbanda and Willemijn F.B. van der Does conducted the study including data collection, data analysis, and data interpretation. Rohit K. Kharbanda and Willemijn F.B. van der Does prepared the manuscript draft with important intellectual input from Natasja M.S. de Groot and Ad J.J.C. Bogers. Lianne N. van Staveren and Yannick J.H.J. Taverne assisted with data interpretation and writing the manuscript. All authors have reviewed the final version of the manuscript and given their approval for submission.

\section{How to Cite This Article}

Kharbanda R.K., van der Does W.F.B., van Staveren L.N., Taverne Y.J.H.J., Bogers A.J.J.C., de Groot N.M.S. 2022. Vagus Nerve Stimulation and Atrial Fibrillation: Revealing the Paradox. Neuromodulation 2022; 25: 356-365.

\section{REFERENCES}

1. Herring N, Kalla M, Paterson DJ. The autonomic nervous system and cardiac arrhythmias: current concepts and emerging therapies. Nat Rev Cardiol 2019:16:707-726.

2. Goldberger JJ, Arora R, Buckley U, Shivkumar K. Autonomic nervous system dysfunction: JACC focus seminar. J Am Coll Cardiol. 2019;73:1189-1206.

3. Stavrakis S, Kulkarni K, Singh JP, Katritsis DG, Armoundas AA. Autonomic modulation of cardiac arrhythmias: methods to assess treatment and outcomes. JACC Clin Electrophysiol. 2020;6:467-483.

4. Shen MJ, Zipes DP. Role of the autonomic nervous system in modulating cardiac arrhythmias. Circ Res. 2014;114:1004-1021.

5. Floras JS. Sympathetic nervous system activation in human heart failure: clinical implications of an updated model. J Am Coll Cardiol. 2009;54:375-385.

6. Dobrev D, Aguilar M, Heijman J, Guichard JB, Nattel S. Postoperative atrial fibrillation: mechanisms, manifestations and management. Nat Rev Cardiol. 2019;16:417-436.

7. Hoffman BF, Siebens AA, Brooks CM. Effect of vagal stimulation on cardiac excitability. Am J Physiol. 1952;169:377-383.

8. Alessi R, Nusynowitz M, Abildskov JA, Moe GK. Nonuniform distribution of vaga effects on the atrial refractory period. Am J Physiol. 1958:194:406-410.

9. Tai CT, Chiou CW, Wen ZC, et al. Effect of phenylephrine on focal atrial fibrillation originating in the pulmonary veins and superior vena cava. J Am Coll Cardiol. 2000:36:788-793.

10. Takei M, Tsuboi M, Usui $T$, et al. Vagal stimulation prior to atrial rapid pacing protects the atrium from electrical remodeling in anesthetized dogs. Jpn Circ J. $2001 ; 65: 1077-1081$

11. Zhang Y, Ilsar I, Sabbah HN, Ben David T, Mazgalev TN. Relationship between right cervical vagus nerve stimulation and atrial fibrillation inducibility: therapeutic intensities do not increase arrhythmogenesis. Heart Rhythm. 2009;6:244-250.

12. Stavrakis $S$, Humphrey MB, Scherlag B, et al. Low-level vagus nerve stimulation suppresses post-operative atrial fibrillation and inflammation: a randomized study. JACC Clin Electrophysiol. 2017;3:929-938.

13. Stavrakis $\mathrm{S}$, Humphrey MB, Scherlag BJ, et al. Low-level transcutaneous electrical vagus nerve stimulation suppresses atrial fibrillation. I Am Coll Cardiol 2015;65:867-875

14. Andreas M, Arzl P, Mitterbauer $A$, et al. Electrical stimulation of the greater auricular nerve to reduce postoperative atrial fibrillation. Circ Arrhythm Electrophysiol. 2019;12:e007711.

15. Stavrakis S, Stoner JA, Humphrey MB, et al. TREAT AF (Transcutaneous Electrical Vagus Nerve Stimulation to Suppress Atrial Fibrillation): a randomized clinical trial. JACC Clin Electrophysiol. 2020;6:282-291.

16. Armour JA. Functional anatomy of intrathoracic neurons innervating the atria and ventricles. Heart Rhythm. 2010;7:994-996.
17. Armour JA, Murphy DA, Yuan BX, Macdonald S, Hopkins DA. Gross and microscopic anatomy of the human intrinsic cardiac nervous system. Anat Rec. 1997;247:289-298.

18. Pauza DH, Skripka V, Pauziene N, Stropus R. Morphology, distribution, and variability of the epicardiac neural ganglionated subplexuses in the human heart. Anat Rec. 2000;259:353-382.

19. Ardell JL, Andresen MC, Armour JA, et al. Translational neurocardiology: preclinical models and cardioneural integrative aspects. J Physiol. 2016:594:3877-3909.

20. Cardinal R, Pagé $P$, Vermeulen M, Ardell JL, Armour JA. Spatially divergent cardiac responses to nicotinic stimulation of ganglionated plexus neurons in the canine heart. Auton Neurosci. 2009;145:55-62.

21. Furukawa Y, Wallick DW, Carlson MD, Martin PJ. Cardiac electrical responses to vagal stimulation of fibers to discrete cardiac regions. Am J Physiol. 1990:258:H1112-H1118.

22. Furukawa $Y$, Hoyano $Y$, Chiba S. Parasympathetic inhibition of sympathetic effects on sinus rate in anesthetized dogs. Am J Physiol. 1996;271:H44-H50.

23. McGuirt AS, Schmacht DC, Ardell JL. Autonomic interactions for control of atrial rate are maintained after SA nodal parasympathectomy. Am J Physiol. 1997;272:H2525-H2533.

24. Groves DA, Brown VJ. Vagal nerve stimulation: a review of its applications and potential mechanisms that mediate its clinical effects. Neurosci Biobehav Rev. 2005;29:493-500

25. Kawashima T. The autonomic nervous system of the human heart with special reference to its origin, course, and peripheral distribution. Anat Embryol (Berl). 2005;209:425-438.

26. Hopkins DA, Armour JA. Ganglionic distribution of afferent neurons innervating the canine heart and cardiopulmonary nerves. J Auton Nerv Syst. 1989:26:213-222.

27. Silvani A, Calandra-Buonaura G, Dampney RA, Cortelli P. Brain-heart interactions: physiology and clinical implications. Philos Trans A Math Phys Eng Sci. 2016;374:20150181.

28. Smeets JL, Allessie MA, Lammers WJ, Bonke Fl, Hollen J. The wavelength of the cardiac impulse and reentrant arrhythmias in isolated rabbit atrium. The role of heart rate, autonomic transmitters, temperature, and potassium. Circ Res. 1986;58:96-108.

29. Scherf D. Chick FB. Abnormal cardiac rhythms caused by acetylcholine. Circulation. 1951:3:764-769.

30. Loomis TA, Krop S. Auricular fibrillation induced and maintained in animals by acetylcholine or vagal stimulation. Circ Res. 1955:3:390-396.

31. Armour JA, Hageman GR, Randall WC. Arrhythmias induced by local cardiac nerve stimulation. Am J Physiol. 1972;223:1068-1075.

32. Hageman GR, Randall WC, Armour JA. Direct and reflex cardiac bradydysrhythmias from small vagal nerve stiumaltions. Am Heart J. 1975;89:338-348.

33. Yelich MR, Euler DE, Wehrmacher WH, Sinha SN, Randall WC. Parasympathetic influence on atrial vulnerability in the puppy. Am J Physiol. 1978;235:H683-H689.

34. Sarmast $F$, Kolli $A$, Zaitsev $A$, et al. Cholinergic atrial fibrillation: I(K,ACh) gradients determine unequal left/right atrial frequencies and rotor dynamics. Cardiovasc Res. 2003;59:863-873.

35. Arora $\mathrm{R}, \mathrm{Ng} \mathrm{J}$, Ulphani J, et al. Unique autonomic profile of the pulmonary veins and posterior left atrium. J Am Coll Cardiol. 2007:49:1340-1348.

36. Haïssaguerre M, Jaïs $P$, Shah DC, et al. Spontaneous initiation of atrial fibrillation by ectopic beats originating in the pulmonary veins. N Engl J Med. 1998;339:659-666.

37. Baykaner $T$, Zografos $T A$, Zaman JAB, et al. Spatial relationship of organized rotational and focal sources in human atrial fibrillation to autonomic ganglionated plexi. Int J Cardiol. 2017;240:234-239.

38. Tan AY, Chen PS, Chen LS, Fishbein MC. Autonomic nerves in pulmonary veins. Heart Rhythm. 2007;4(suppl 3):S57-S60.

39. Patterson E, Lazzara R, Szabo B, et al. Sodium-calcium exchange initiated by the Ca2+ transient: an arrhythmia trigger within pulmonary veins. J Am Coll Cardiol. 2006:47:1196-1206.

40. Patterson E, Po SS, Scherlag BJ, Lazzara R. Triggered firing in pulmonary veins initiated by in vitro autonomic nerve stimulation. Heart Rhythm. 2005:2:624-631.

41. Chen YJ, Chen SA, Chang MS, Lin Cl. Arrhythmogenic activity of cardiac muscle in pulmonary veins of the dog: implication for the genesis of atrial fibrillation. Cardiovasc Res. 2000;48:265-273.

42. Coumel P. Autonomic influences in atrial tachyarrhythmias. I Cardiovasc Electrophysiol. 1996;7:999-1007.

43. Chen PS, Tan AY. Autonomic nerve activity and atrial fibrillation. Heart Rhythm 2007:4(suppl 3):S61-S64

44. Chen PS, Chen LS, Fishbein MC, Lin SF, Nattel S. Role of the autonomic nervous system in atrial fibrillation: pathophysiology and therapy. Circ Res. 2014;114:1500-1515.

45. Wang J, Liu L, Feng J, Nattel S. Regional and functional factors determining induction and maintenance of atrial fibrillation in dogs. Am J Physiol. 1996;271:H148-H158.

46. Rensma PL, Allessie MA, Lammers WJ, Bonke Fl, Schalij MJ. Length of excitation wave and susceptibility to reentrant atrial arrhythmias in normal conscious dogs. Circ Res. 1988;62:395-410.

47. Liu L, Nattel S. Differing sympathetic and vagal effects on atrial fibrillation in dogs: role of refractoriness heterogeneity. Am J Physiol. 1997;273H805-H816.

48. Vigmond EJ, Tsoi V, Kuo S, et al. The effect of vagally induced dispersion of action potential duration on atrial arrhythmogenesis. Heart Rhythm. 2004;1:334-344.

49. Schwartz PJ, De Ferrari GM, Sanzo A, et al. Long term vagal stimulation in patients with advanced heart failure: first experience in man. Eur J Heart Fail. 2008:10:884891.

50. Stavrakis S, Scherlag BJ, Fan Y, et al. Antiarrhythmic effects of vasostatin-1 in a canine model of atrial fibrillation. J Cardiovasc Electrophysiol. 2012;23:771-777. 
51. Stavrakis S, Scherlag BJ, Fan Y, et al. Inhibition of atrial fibrillation by low-leve vagus nerve stimulation: the role of the nitric oxide signaling pathway. J Interv Card Electrophysiol. 2013;36:199-208.

52. Xi Y, Cheng J. Dysfunction of the autonomic nervous system in atrial fibrillation. J Thorac Dis. 2015;7:193-198.

53. Yang $D, X i$ Y, Ai T, et al. Vagal stimulation promotes atrial electrical remodeling induced by rapid atrial pacing in dogs: evidence of a noncholinergic effect. Pacing Clin Electrophysiol. 2011;34:1092-1099.

54. Liu Y, Scherlag BJ, Fan Y, et al. Inducibility of atrial fibrillation after GP ablations and "autonomic blockade": evidence for the pathophysiological role of the nonadrenergic and noncholinergic neurotransmitters. J Cardiovasc Electrophysiol. 2013;24:188-195.

55. Henning RJ, Sawmiller DR. Vasoactive intestinal peptide: cardiovascular effects. Cardiovasc Res. 2001;49:27-37.

56. Li S, Scherlag BJ, Yu L, et al. Low-level vagosympathetic stimulation: a paradox and potential new modality for the treatment of focal atrial fibrillation. Circ Arrhythm Electrophysiol. 2009;2:645-651.

57. Yu L, Scherlag BJ, Sha Y, et al. Interactions between atrial electrical remodeling and autonomic remodeling: how to break the vicious cycle. Heart Rhythm. 2012:9:804-809.

58. Shen MJ, Shinohara T, Park HW, et al. Continuous low-level vagus nerve stimulation reduces stellate ganglion nerve activity and paroxysmal atrial tachyarrhythmias in ambulatory canines. Circulation. 2011;123:2204-2212.

59. Sheng $X$, Scherlag $B J, Y u L$, et al. Prevention and reversal of atrial fibrillation inducibility and autonomic remodeling by low-level vagosympathetic nerve stimulation. J Am Coll Cardiol. 2011;57:563-571.

60. Sha Y, Scherlag BJ, Yu L, et al. Low-level right vagal stimulation: anticholinergic and antiadrenergic effects. J Cardiovasc Electrophysiol. 2011;22:1147-1153.

61. Shen MJ, Chang Hao-Che, Park HW, et al. Low-level vagus nerve stimulation upregulates small conductance calcium-activated potassium channels in the stellate ganglion. Heart Rhythm. 2013;10:910-915.

62. Cho Y, Cha MJ, Choi EK, Oh IY, Oh S. Effects of low-intensity autonomic nerve stimulation on atrial electrophysiology. Korean Circ J. 2014:44:243-249.

63. Yuan $Y$, Jiang $Z, H e Y$, et al. Continuous vagal nerve stimulation affects atrial neura remodeling and reduces atrial fibrillation inducibility in rabbits. Cardiovasc Pathol. 2015;24:395-398.

64. Lu Y, Sun J, Zhou X, Zhang L, Ma M, Tang B. Effect of low-level vagus nerve stimulation on cardiac remodeling in a rapid atrial pacing-induced canine model of atrial fibrillation. J Cardiovasc Pharmacol. 2016;67:218-224.

65. Yu L, Scherlag BJ, Li S, et al. Low-level transcutaneous electrical stimulation of the auricular branch of the vagus nerve: a noninvasive approach to treat the initia phase of atrial fibrillation. Heart Rhythm. 2013;10:428-435.

66. Chen $\mathrm{M}, \mathrm{Yu} \mathrm{L}$, Liu $\mathrm{Q}$, et al. Low level tragus nerve stimulation is a non-invasive approach for anti-atrial fibrillation via preventing the loss of connexins. Int J Cardiol. 2015;179:144-145.

67. Chen $M$, Zhou $X$, Liu $\mathrm{O}$, et al. Left-sided noninvasive vagus nerve stimulation suppresses atrial fibrillation by upregulating atrial gap junctions in canines. J Cardiovasc Pharmacol. 2015;66:593-599.

68. Salavatian S, Beaumont E, Longpré JP, et al. Vagal stimulation targets select populations of intrinsic cardiac neurons to control neurally induced atrial fibrillation. Am J Physiol Heart Circ Physiol. 2016;311:H1311-H1320.

69. Clancy JA, Mary DA, Witte KK, Greenwood JP, Deuchars SA, Deuchars J. Noninvasive vagus nerve stimulation in healthy humans reduces sympathetic nerve activity. Brain Stimul. 2014;7:871-877.

70. Park JH, Pak HN, Lee S, Park HK, Seo JW, Chang BC. The clinical significance of the atrial subendocardial smooth muscle layer and cardiac myofibroblasts in human atrial tissue with valvular atrial fibrillation. Cardiovasc Pathol. 2013:22:58-64.

71. Beaumont E, Southerland EM, Hardwick JC, et al. Vagus nerve stimulation mitigates intrinsic cardiac neuronal and adverse myocyte remodeling postmyocardial infarction. Am J Physiol Heart Circ Physiol. 2015;309:H1198-H1206.
72. Beaumont $\mathrm{E}$, Wright $\mathrm{GL}$, Southerland $\mathrm{EM}$, et al. Vagus nerve stimulation mitigates intrinsic cardiac neuronal remodeling and cardiac hypertrophy induced by chronic pressure overload in guinea pig. Am J Physiol Heart Circ Physiol. 2016;310:H1349_ H1359.

73. Rossi P, Ricci A, De Paulis R, et al. Epicardial ganglionated plexus stimulation decreases postoperative inflammatory response in humans. Heart Rhythm. 2012;9:943-950.

74. Pavlov VA, Tracey KJ. The vagus nerve and the inflammatory reflex-linking immunity and metabolism. Nat Rev Endocrinol. 2012;8:743-754.

75. Xiong J, Wang $H$, Bao $Y$, Guo $Y$, Sun $Y$. Electric vagal nerve stimulation inhibits inflammation and improves early postoperation cognitive dysfunction in aged rats. BMC Anesthesiol. 2019;19:217.

76. Peuker ET, Filler TJ. The nerve supply of the human auricle. Clin Anat. 2002;15:3537.

77. Burger AM, Verkuil B. Transcutaneous nerve stimulation via the tragus: are we really stimulating the vagus nerve? Brain Stimul. 2018;11:945-946.

78. Badran BW, Dowdle LT, Mithoefer OJ, et al. Neurophysiologic effects of trans cutaneous auricular vagus nerve stimulation (taVNS) via electrical stimulation of the tragus: a concurrent taVNS/fMRI study and review. Brain Stimul. 2018;11:492500.

79. Seki A, Green HR, Lee TD, et al. Sympathetic nerve fibers in human cervical and thoracic vagus nerves. Heart Rhythm. 2014;11:1411-1417.

80. Yamakawa K, Rajendran PS, Takamiya $T$, et al. Vagal nerve stimulation activates vagal afferent fibers that reduce cardiac efferent parasympathetic effects. Am Physiol Heart Circ Physiol. 2015;309:H1579-H1590.

81. McAllen RM, Shafton AD, Bratton BO, Trevaks D, Furness JB. Calibration of thresholds for functional engagement of vagal $A, B$ and $C$ fiber groups in vivo. Bioelectron Med (Lond). 2018;1:21-27.

82. Yoo PB, Liu H, Hincapie JG, Ruble SB, Hamann JJ, Grill WM. Modulation of heart rate by temporally patterned vagus nerve stimulation in the anesthetized dog. Physiol Rep. 2016;4:12689.

83. Vonck KEJ, Larsen LE. Neuromodulation: Comprehensive Textbook of Principles, Technologies, and Therapies. 2nd ed. Academic Press: 2018:211-221.

84. Qing KY, Wasilczuk KM, Ward MP, et al. B fibers are the best predictors of cardiac activity during vagus nerve stimulation: Qing, vagal B fiber activation and cardiac effects. Bioelectron Med. 2018:4:5

85. Kaniusas E, Kampusch S, Tittgemeyer M, et al. Current directions in the auricular vagus nerve stimulation $I 1-a n$ engineering perspective. Front Neurosci. 2019;13:772.

86. Napadow V, Edwards RR, Cahalan CM, et al. Evoked pain analgesia in chronic pelvic pain patients using respiratory-gated auricular vagal afferent nerve stimulation. Pain Med. 2012;13:777-789.

\section{COMMENT}

This review provides an excellent overview of the preclinical and clinical literature with respect to control of the atrial arrhythmia potential as affected by focal vagal nerve stimulation. It highlights important aspects of efficacy as affected by site of delivery and stimulation protocol. Likewise, it considers critical mechanisms affected by $\mathrm{AF}$ and VNS, including changes in the neural and electrophysiological substrates as well as the effects on focal cardiac immune responses.

Jeffrey Ardell, PhD Los Angeles, CA, USA 